\title{
Hidratación oral con una solución de baja osmolaridad en niños deshidratados por enfermedades diarreicas: un estudio clínico controlado
}

\author{
Carlos Bernal ${ }^{1}$, Claudia Velásquez ${ }^{2}$, Guillermo García ${ }^{1}$ \\ Gustavo Uribe ${ }^{3}$, Carlos Mauricio Palacio ${ }^{5}$ \\ ${ }^{1}$ Departamento de Pediatría y Puericultura, Universidad de Antioquia, Medellín, Colombia. \\ ${ }^{2}$ Escuela de Nutrición y Dietética, Universidad de Antioquia, Medellín, Colombia. \\ ${ }^{3}$ Hospital Universitario San Vicente de Paúl, Medellín, Colombia. \\ ${ }^{5}$ Universidad de Antioquia, Medellín, Colombia.
}

Se realizó un estudio clínico controlado para comparar la efectividad de un suero de baja osmolaridad (SBO) (245 mOsm/L), en relación con la solución de hidratación oral estándar recomendada por la OMS (SHO/OMS). El grupo 1 (69 niños) recibió el suero de hidratación oral, SHO/90 (311 mOsml/L) y el grupo 2 (71 niños) el suero de baja osmolaridad, SBO/75 (245 $\mathrm{mOsm} / \mathrm{L})$. La hidratación se consiguió con éxito en $88,4 \%$ en el grupo 1 y en $92,9 \%$ en el grupo $2(p=0,35)$. El tiempo de hidratación fue 5,2 horas $(D E=1,8)$ en el grupo 1 y $5,5 \quad(D E=1,7)$ en grupo $2(p=0,31)$. El gasto fecal fue $6,3 \mathrm{~g} / \mathrm{kg} / \mathrm{hora}(D E=5,0)$ en el grupo $1 \mathrm{y} 5,6 \mathrm{~g} / \mathrm{kg} / \mathrm{hora}$ $(D E=5,1)$ en el grupo $2(p=0,94)$. El sodio al final de la hidratación fue $139,3 \mathrm{mEq} / \mathrm{L}(\mathrm{DE}=7,1)$ en el grupo 1 y $136,7 \mathrm{mEq} / \mathrm{L}(\mathrm{DE}=4,3)$ en el grupo $2(p=0,014)$. El período de observación fue de 21 horas $(D E=5,7)$ en el grupo 1 y de 22 horas $(D E=5,6)$ en el grupo 2 . El gasto fecal en el grupo 1 fue 5,2 g/kg/hora ( $D E=4,1)$ y $4,2 \mathrm{~g} / \mathrm{kg} / \mathrm{hora}(\mathrm{DE}=4,1)$ en el grupo $2(\mathrm{p}=0,16)$. Necesitaron líquidos endovenosos $23,1 \%$ en el grupo 1 y $9,8 \%$ en el grupo $2(p=0,033)$. La solución de baja osmolaridad tiene la ventaja de disminuir la necesidad de utilizar líquidos endovenosos, corrige la mayor parte de los trastornos del sodio plasmático en niños deshidratados y no produce riesgo de desarrollo de hiponatremia.

Palabras clave: deshidratación, diarrea, diarrea infantil, rehidratación oral, soluciones para rehidratación, terapia con líquidos.

Effectiveness of oral hydration therapy with a low osmolarity solution in children dehydrated by diarrhea

A clinical trial was conducted to compare the efficacy of a low-osmolarity solution ( $245 \mathrm{mOsm} /$ $\mathrm{L})$, and a standard oral rehydration solution (ORS) recommended by WHO for children dehydrated by diarrhea. Group 1 (69 children) received WHO/ORS (311 mOsml/L) and group 2 (71 children) received a low-osmolarity solution $(245 \mathrm{mOsm} / \mathrm{L})$. Rehydration was successful in $88.4 \%$ in group 1 and $92.9 \%$ in group $2(p=0.35)$. Rehydration was completed in $5.2 \mathrm{~h}(\mathrm{SD}+1$ - 1.8) in group 1 and 5.5 (SD +/- 1.7) in group $2(p=0.31)$. Stool output was $6.3 \mathrm{~g} / \mathrm{kg} / \mathrm{h}(\mathrm{SD}+/-5.0)$ in group 1 and $5.6 \mathrm{~g} / \mathrm{kg} / \mathrm{h}(S D+/-5.1)$ in group $2(p=0.94)$. Sodium at rehydration-completion was $139.3 \mathrm{mEq} / \mathrm{L}(\mathrm{SD}+/-7.1)$ in group 1 and $136.7 \mathrm{mEq} / \mathrm{L}(\mathrm{SD}+/-4.3)$ in group $2(p=0.014)$. Group 1 was under observation for 21 hours (SD +/- 5.7) and group 2, for 22 hours (SD +/- 5.6). Stool output in group 1 was $5.2 \mathrm{~g} / \mathrm{kg} / \mathrm{h}$ (SD 4.1) and $4.2 \mathrm{gr} . / \mathrm{kg} / \mathrm{h}(\mathrm{SD}+/-4.1)$ in group $2(p=0.16)$. In group 1, 23.1\% required intravenous solutions and $9.8 \%$ in group $2(p=0.03)$. In treating dehydrated children, the low-osmolarity solution diminished the need for intravenous solutions, corrected most plasmatic sodium disorders, and produced no-risk of developing hyponatremia.

Key words: dehydration, diarrhea, infantile diarrhea, rehydration, fluid therapy, oral rehydration therapy. 
La hidratación oral es uno de los descubrimientos médicos más importantes del siglo XX; su base fisiológica es el transporte acoplado de sodio con otros solutos, lo cual favorece el ingreso de agua a las células del intestino.

Después de muchos estudios, a partir de 1975, la Organización Mundial de la Salud (OMS) recomendó una fórmula de suero oral con la siguiente composición en $\mathrm{mmol} / \mathrm{L}$ : sodio: 90 , cloro 80 , potasio 20, bicarbonato 30 (a partir de 1984 el bicarbonato fue reemplazado por citrato trisódico, $10 \mathrm{mmol} / \mathrm{L}$ ) y glucosa 111 . Inicialmente, la osmolaridad era 331 , a partir del cambio del bicarbonato por citrato, la osmolaridad es de 311 $\mathrm{mOsm} / \mathrm{L}$.

Desde el inicio del programa de control de enfermedades diarreicas, la OMS ha mantenido la política de tener un solo suero oral recomendado que sea efectivo para prevenir y tratar la deshidratación producida por la diarrea de cualquier etiología, incluso el cólera.

No obstante ser un remedio maravilloso, el suero oral con la fórmula recomendada tiene limitaciones: no disminuye ni el volumen de las evacuaciones ni el tiempo de duración de la diarrea.

Desde hace años se empezó a buscar una fórmula que fuera mejor. Se inició la búsqueda del 'super suero': un suero que, además de hidratar y prevenir la deshidratación, disminuyera el volumen de las evacuaciones y el tiempo de duración de la diarrea y que, idealmente, contribuyera a la nutrición.

La búsqueda de una solución mejorada ha incluido estudios con aminoácidos, con arroz, con otros cereales, con harina de plátano, con maltodextrinas, con hidrolizados de proteínas, con

Este estudio obtuvo el primer premio en la $\mathrm{V}$ Bienal de Pediatría, Colsubisido, Américas, y fue publicado en la revista Saludarte 2000;1:6-23. Se publica con autorización de la revista Saludarte.

Correspondencia:

Carlos Bernal, Departamento de Pediatría y Puericultura, Universidad de Antioquia. Apartado aéreo 1226, Medellín. Teléfono: 263 7885; Fax 2637885.

cabp @ epm.net.co

Recibido: 27/11/02; aceptado: 25/02/03 amilasas y con soluciones de baja osmolaridad (1-12).

Solamente las soluciones similares al suero de hidratación oral con $90 \mathrm{mmol} / \mathrm{L}$ de sodio (SHO/ $90)$, pero que en lugar de tener $20 \mathrm{~g} / \mathrm{L}$ de glucosa, contienen 50 a $80 \mathrm{~g} / \mathrm{L}$ de polvo de arroz, demostraron ser superiores para el tratamiento de los pacientes con cólera (2).

El haber observado en algunos pacientes con diarrea no colérica que el volumen fecal aparentemente aumenta con la utilización del SHO/ 90 , motivó la idea de que, siendo éste ligeramente hiperosmolar, pudiera en algunos pacientes agravar una situación de diarrea osmótica; en estos casos sería posible obtener mejores resultados con soluciones con una osmolaridad menor. Se realizó un estudio en el cual se comparó el SHO/90 con una mezcla en la cual un sobre de suero oral se diluía en 1,5 litros de agua, resultando en la siguiente composición en $\mathrm{mmol} /$ L: glucosa 75 , sodio 60 , potasio 13 , cloro 53 , citrato 6,6 , con una osmolaridad de $210 \mathrm{mOsm} /$ L. En el estudio se encontró que el gasto fecal fue significativamente menor en los niños que recibieron la solución diluída, así como una disminución no significativa de los niveles plasmáticos de sodio entre los niños que recibieron la solución diluída. En este estudio la muestra fue relativamente pequeña (11).

Posteriormente, se realizó un estudio multicéntrico (12) en el cual se incluyeron 447 niños deshidratados por enfermedad diarreica aguda, los cuales fueron asignados en forma aleatoria para recibir bien fuera el SHO/90 o una SBO (224 $\mathrm{mOsm} / \mathrm{L}$ ) con la siguiente composición en $\mathrm{mmol} /$ L: glucosa 84 , sodio 60 , potasio 20 , cloro 50 y citrato 10 . El gasto fecal fue $39 \%$ mayor y la duración de la diarrea fue $22 \%$ más prolongada en el grupo de niños que recibió el SHO/90. Así mismo, el riesgo de requerir hidratación intravenosa, después de haberse conseguido la hidratación oral también fue más elevado. Sin embargo, entre los niños que recibieron la solución con $60 \mathrm{mmol} / \mathrm{L}$ de sodio, se encontró una disminución significativa del sodio plasmático, aun cuando no se presentaron casos de hiponatremia clínica. 
En 1994, la OMS recomendó hacer nuevos estudios con una solución con la siguiente composición: sodio $75 \mathrm{mmol} / \mathrm{L}$, glucosa $75 \mathrm{mmol} /$ $\mathrm{L}$, potasio $20 \mathrm{mmol} / \mathrm{L}$, cloro $65 \mathrm{mmol} / \mathrm{L}$, citrato trisódico $10 \mathrm{mmol} / \mathrm{L}$ y osmolaridad de $245 \mathrm{mOsm} /$ L (13).

El primer estudio con esta solución fue realizado por Santosham et al. (14), quienes encontraron que el gasto fecal entre los niños que recibieron el suero de baja osmolaridad fue $36 \%$ menor durante el periodo de hidratación y que la posibilidad de necesitar hidratación intravenosa durante el periodo de observación fue significativamente menor entre los niños que recibieron la SBO. Se concluyó que el uso de la SBO tuvo efectos benéficos en el curso clínico de niños con diarrea aguda por la disminución del gasto fecal, por la disminución de la proporción de niños con vómito durante la fase de rehidratación y por la menor necesidad de suplementar con terapia intravenosa.

El estudio que se presenta en esta publicación utilizó la misma solución, con la diferencia de que fue preparada en forma líquida por la planta de sueros de Corpaúl, Medellín. Su objetivo fue comparar la efectividad de una SBO en relación con el SHO/90 para la hidratación oral de niños deshidratados por diarrea, incluidos niños deshidratados por diarreas agudas, persistentes o disentéricas, en niños eutróficos o desnutridos.

Los resultados de este estudio fueron publicados en la revista Saludarte en el 2000 (15). Después de esta publicación han aparecido otros estudios y han ocurrido hechos tan importantes como que la OMS cambió oficialmente la formulación del suero oral por el de baja osmolaridad. En este artículo se presentan nuevamente los resultados y se discuten los hechos ocurridos en los últimos dos años.

\section{Materiales y métodos}

\section{Diseño del estudio}

Se diseñó un estudio clínico controlado triple ciego. La población la constituyeron niños con edades comprendidas entre 1 y 36 meses que presentaban diarrea de menos de un mes de evolución, con deshidratación no acompañada de choque hipovolémico.

El estudio se realizó en la Unidad de Investigación, Capacitación y Tratamiento de la Diarrea del Hospital Universitario San Vicente de Paúl de Medellín entre el 1 de febrero de 1998 y el 28 de febrero de 1999. Fue aprobado por el Comité de Ética del Hospital Universitario San Vicente de Paúl y el Centro de Investigaciones Médicas de la Universidad de Antioquia.

\section{Cálculo del tamaño de muestra}

La variable utilizada para calcular el tamaño de la muestra fue el gasto fecal durante las primeras veinticuatro horas. En el estudio de Guiraldes, realizado en Chile, citado en el metanálisis sobre los sueros a base de arroz (2), en pacientes similares a los incluidos en este estudio, el gasto fecal durante las primeras 24 horas en niños que recibían la SHO/90 fue $126 \mathrm{~g} / \mathrm{kg}(\mathrm{DE}=64)$. Se consideró deseable una disminución de $25 \%$ en el gasto fecal entre los niños que recibieran la solución experimental en relación con la SHO/90. Para calcular el tamaño de la muestra, se utilizó la fórmula recomendada por Pocock (16), aceptando un error tipo I de 0,05 y un error tipo II igual a 0,20 . Se necesitaban 66 pacientes en cada grupo, finalmente se incluyeron 69 en el grupo 1 que recibieron $\mathrm{SHO} / 90$ y 71 en el grupo 2 que recibieron la solución de baja osmolaridad (SBO/ 75).

\section{Criterios de inclusión}

El estado de hidratación se evaluó de acuerdo con los parámetros establecidos por el programa Control de Enfermedades Diarreicas (CED) de la OMS (17). En los niños que presentaban deshidratación sin choque hipovolémico, se evaluó la presencia de los siguientes criterios de inclusión:

- niños de sexo masculino ${ }^{1}$ con edades entre 1 y 36 meses;

- diarrea con una duración menor de un mes, acompañada de deshidratación sin choque hipovolémico;

\footnotetext{
${ }^{1}$ No se incluyeron las niñas por la dificultad en la medición precisa de la eliminación urinaria.
} 
- historia de haber presentado, por lo menos, tres deposiciones líquidas o semilíquidas en las veinticuatro horas anteriores al ingreso;

- ausencia de otras enfermedades graves asociadas, y

- aceptación de la familia.

\section{Criterios de exclusión}

Se excluyeron los niños que presentaban los siguientes criterios:

- niños con desnutrición edematosa (kwashiorkor);

- niños que hubieran participado antes en el estudio y que volvieron deshidratados, y

- niños que presentaran al ingreso cuadro clínico sospechoso de íleo paralítico.

\section{Aleatorización}

Una vez verificados los criterios de inclusión y obtenido el consentimiento informado por parte de los padres del niño, se procedió a la aleatorización. En el Centro de Investigaciones de la Facultad de Medicina, personas diferentes a los investigadores prepararon una lista maestra de aleatorización utilizando bloques permutados de longitud variable, entre 8 y 12 por grupo. Con base en la lista maestra, se prepararon sobres cerrados de papel opaco, numerados en forma consecutiva. En los sobres se incluyó el nombre del suero que debería recibir el niño. Las personas encargadas de preparar el suero abrían los sobres, preparaban el suero indicado y lo envasaban en frascos marcados con el número correspondiente al estudio. La apariencia física de ambos sueros era similar.

\section{Período de hidratación}

Se consideró como período de hidratación al tiempo transcurrido entre el ingreso y el momento en que el niño se hidrató.

Llevada a cabo la aleatorización, los niños fueron pesados y medidos, se inició la hidratación y se evaluaron cada hora.

A los niños se les ofreció entre 50 y $100 \mathrm{ml} / \mathrm{kg}$ durante las primeras cuatro a seis horas, pero la cantidad fue mayor o menor a libre demanda, utilizando el volumen necesario hasta conseguir la hidratación. Las soluciones fueron administradas con vaso y cuchara en forma continua. La persona encargada de administrar las soluciones fue la madre, el padre o el acompañante de los niños, bajo la supervisión y apoyo continuo del personal médico y de enfermería contratado para el estudio. En caso de vómito, se suspendía la administración de la solución durante cinco minutos y se continuaba ofreciendo el suero más lentamente. Si el vómito persistía se utilizaba sonda nasogástrica. La sonda se utilizó también en casos de diarrea de alto gasto o cuando a pesar de la deshidratación el niño no recibía la solución por rechazo al suero oral o por cansancio físico. La velocidad a la cual se administró el suero a través de la sonda nasogástrica fue entre 20 y $25 \mathrm{ml} / \mathrm{kg} /$ hora. Se consideró éxito cuando la hidratación se consiguió por vía oral o por sonda con $\mathrm{SHO}$ en un tiempo máximo de 10 horas. Los niños que no pudieron hidratarse por vía oral ni por sonda nasogástrica en un periodo de diez horas, recibieron hidratación parenteral: estos casos se consideraron fracasos.

El peso se registró al ingreso y cuando se consideró que el niño estaba hidratado. La clasificación del riesgo nutricional se realizó después de conseguida la hidratación de acuerdo con las normas del National Center for Health Statistics (NCHS) (20). Se utilizó el indicador de desnutrición aguda peso para la talla $(p / t)$. El análisis se hizo mediante el puntaje $Z$ con base en el programa Epinut. El punto de corte de este indicador fue de una desviación estándar.

\section{Exámenes de laboratorio}

En los primeros minutos después de iniciada la hidratación se tomó una muestra de sangre para determinar concentraciones de sodio y potasio. También se tomaron, cuando fue posible, muestras de materias fecales para coprológico, coprocultivo y rotavirus. La muestra se tomó por defecación espontánea o provocada por estímulo rectal con termómetro, directamente en un recipiente limpio y se envió inmediatamente al laboratorio del Servicio de Infectados del Hospital Infantil, donde se hicieron los siguientes procedimientos: observación al microscopio de un extendido de la muestra en solución salina para buscar trofozoítos y quistes de protozoos, huevos o larvas de helmintos, esporas y psedomicelios 
de hongos, leucocitos, eritrocitos y grasas; tinción de Ziel Nielsen modificado para Cryptosporidium sp.; tinción de Gram modificada para Campylobacter sp.; siembra en los siguientes medios de cultivo: MacConkey, SS, Heptoen, XLD, medio para Yersinia y TCBS.

La identificación de los rotavirus se hizo mediante una prueba de látex Murex (R).

Todos los procedimientos de los cultivos y las tinciones para Cryptosporidium y Campylobacter se hicieron de acuerdo con el manual para investigaciones de laboratorio en niños con diarrea aguda de la OMS (18). Al terminar la hidratación y al final del periodo de observación, se tomaron de nuevo muestras de sangre para medir sodio y potasio.

\section{Periodo de observación}

Se consideró como periodo de observación el tiempo durante el cual los niños recibieron suero oral; para los éxitos, equivalió al tiempo de permanencia en la sala; para los fracasos, tanto tempranos como tardíos, al tiempo durante el cual recibieron suero oral hasta el momento en el que fue necesario administrar líquidos endovenosos.

Una vez hidratados los niños, se continuó la reposición de las pérdidas concomitantes con la misma solución utilizada para la hidratación y se inició la alimentación con una dieta estandarizada de acuerdo con la edad. Cuando los niños recibían leche materna, ésta se continuó. A los niños menores de seis meses que no recibían leche materna se les ofreció $150 \mathrm{ml} / \mathrm{kg} /$ día de una fórmula libre de lactosa preparada al 13\%, fraccionada en ocho tomas. Los niños mayores de seis meses recibieron $100 \mathrm{ml} / \mathrm{kg} /$ día de la misma fórmula libre de lactosa repartida en seis tomas y, además, dos sopas diarias. El tratamiento de los pacientes después de las primeras veinticuatro horas se continuó según su evolución de acuerdo con los protocolos establecidos en el Departamento de Pediatría de la Universidad de Antioquia (19).

Los niños fueron pesados nuevamente al terminar el periodo de observación.

Se consideró fracaso tardío cuando los niños volvieron a deshidratarse y necesitaron líquidos parenterales durante el período de observación. Los pacientes que fueron retirados del estudio por fracaso, por voluntad de sus padres o por cualquier otra complicación de tipo médico se incluyeron en el análisis hasta el momento en que fueron retirados.

\section{Mediciones}

La medición del gasto fecal se hizo mediante el empleo de pañales desechables prepesados. La recolección de orina se hizo mediante bolsas recolectoras. La medición del volumen de los vómitos se hizo mediante el empleo de paños prepesados.

La longitud de los niños se determinó por infantómetro de madera con sensibilidad de $1 \mathrm{~mm}$. El peso se determinó en balanza mecánica Detecto Doctors Infant Scale ${ }^{\circledR}$ con sensibilidad de $5 \mathrm{~g}$.

El peso de los pañales y de los paños utilizados para secar los vómitos se hizo en balanza Ohaus Triple Beam Balance, calibrada de gramo en gramo hasta $500 \mathrm{~g}$.

\section{Procesamiento de la información}

El almacenamiento de los datos se hizo utilizando el programa Epilnfo, versión 6.0.1 (CDC, Atlanta, EE.UU).

Una vez incluida toda la información en la base de datos, una persona diferente a los investigadores y al estadístico, teniendo en cuenta la lista maestra de aleatorización, introdujo un campo para cada uno de los grupos en los formularios, denominándolos grupos uno o dos. De esta manera, el análisis de los resultados en los dos grupos se hizo sin que los investigadores ni el estadístico supieran cuál de los dos grupos correspondía al SHO/90 y cuál al de baja osmolaridad. Esta clave sólo fue revelada a los investigadores en el momento en el que se encontraban listos los resultados y hecho el análisis estadístico.

\section{Análisis estadístico}

Los valores de las variables medidas tanto al ingreso del estudio como al final del período de hidratación y del período de observación, fueron comparados para los dos grupos de niños, usando 
la prueba $t$ de Student de muestras independientes. Las comparaciones dentro de cada grupo se hicieron usando la prueba t pareada.

Las comparaciones de frecuencias o porcentajes en los dos grupos se llevaron a cabo mediante la prueba exacta de Fisher o la generalizada de Freeman-Halton. Se evaluó el riesgo relativo de la necesidad de utilizar líquidos intravenosos.

Las comparaciones y pruebas se consideraron significativas cuando $p<0,05$. Para el análisis de los datos se usaron los paquetes estadísticos StatXact 4.0 y Statistica 98.

\section{Resultados}

Sesentay nueve niños recibieron el suero $\mathrm{SHO} /$ 90 (grupo 1) y 71 la SBO (grupo 2). No se encontraron diferencias estadísticas significativas en las características del ingreso entre ambos grupos (cuadro 1).

Tampoco se presentó diferencia estadística significativa entre los grupos en relación con el estado nutricional. En el grupo 2 hubo dos niños que presentaban peso para la talla por debajo de menos 3 desviaciones estándar, es decir, con desnutrición grave (cuadro 2).

No se presentaron diferencias estadísticas significativas en relación con los agentes productores de diarrea identificados en ambos grupos, excepto con Escherichia coli enteropatógeno, el cual fue identificado en mayor proporción en el grupo 1 ( $p=0,009)$ (cuadro 3).

\section{Periodo de hidratación}

Los datos referentes al periodo de hidratación se presentan en el cuadro 4. La hidratación se consiguió con éxito en 61 niños $(88,4 \%)$ del grupo 1 y en $66(92,9 \%)$ del grupo $2(p=0,35)$.

No fue posible conseguir la hidratación en 8 casos del grupo 1 y en 5 del grupo 2. Estos casos fueron considerados fracasos tempranos, terminaron de hidratarse con solución polielectrolítica por vía intravenosa y se excluyeron del estudio a partir de ese momento.

No se presentaron diferencias estadísticas significativas en relación con el tiempo de hidratación.

El gasto fecal durante el periodo de hidratación se analizó en todos los pacientes, incluidos éxitos $y$ fracasos, hasta el momento en que fue necesario administrar líquidos intravenosos. El promedio del gasto fecal durante el período de hidratación en el grupo 1 fue de $6,3 \mathrm{~g} / \mathrm{kg} / \mathrm{hora}(\mathrm{DE}=5,02)$ y de $5,6 \mathrm{~g} / \mathrm{kg} /$ hora $(D E=5,07)$ para el grupo 2. Estas diferencias no fueron estadísticamente significativas $(p=0,37)$.

El volumen del suero necesario para conseguir la hidratación fue 99,4 ml/kg en el grupo 1 y de 110,3 $\mathrm{ml} / \mathrm{kg}$ en el grupo $2(\mathrm{p}=0,19)$.

La ganancia de peso durante el período de hidratación fue más elevada en el grupo 2 , pero las diferencias no fueron estadísticamente significativas $(p=0,06)$.

Cuadro 1. Características de los niños al ingreso en cada uno de los grupos de estudio.

\begin{tabular}{|c|c|c|c|c|c|}
\hline & \multicolumn{2}{|c|}{$\begin{array}{c}\text { Grupo } 1 \\
\text { SHO/OMS }(n=69)\end{array}$} & \multicolumn{3}{|c|}{$\begin{array}{c}\text { Grupo } 2 \\
\text { Solución de baja osmolaridad }(n=71)\end{array}$} \\
\hline & $X$ & DE & $\mathbf{X}$ & DE & $p$ \\
\hline & 11,52 & 6,03 & 13,09 & 8,77 & 0,21 \\
\hline Días con diarrea & 5,34 & 4,96 & 5,60 & 6,59 & 0,79 \\
\hline Deposiciones al día & 9,73 & 5,71 & 9,60 & 5,99 & 0,90 \\
\hline Diarrea persistente & $9(13,0 \%)$ & $4,1 \%$ & $9(12,7 \%)$ & $3,9 \%$ & 0,94 \\
\hline Sangre en las heces & $7(10,1 \%)$ & $3,6 \%$ & $15(21,0 \%)$ & $21,0 \%$ & 0,083 \\
\hline Historia de vómito & $60(86,9 \%)$ & $4,0 \%$ & $65(91,5 \%)$ & $3,3 \%$ & 0,35 \\
\hline Vómitos por día & 8,10 & 9,29 & 10,15 & 17,43 & 0,41 \\
\hline Antibióticos & $27(39,1 \%)$ & $5,9 \%$ & $24(33,8 \%)$ & $5,6 \%$ & 0,54 \\
\hline Antiparasitarios & $6(8,5 \%)$ & $3,4 \%$ & $9(12,6 \%)$ & $3,9 \%$ & 0,50 \\
\hline Antidiarreicos & $2(2,8 \%)$ & $2,0 \%$ & $4(5,6 \%)$ & $2,7 \%$ & 0,56 \\
\hline Peso al ingreso (gr) & 8035,7 & 1627,0 & 8270,0 & 2021,5 & 0,45 \\
\hline Talla (cm) & 72,4 & 7,67 & 74,00 & 9,96 & 0,29 \\
\hline
\end{tabular}


Cuadro 2. Estado nutricional peso para la talla en unidades $Z$ en cada uno de los grupos de estudio.

Grupo 1

Grupo 2

SHO/OMS $(n=69)$

Solución de baja osmolaridad $(n=71)$

Exceso de peso, mayor de una DE

Normal, entre $-1 \mathrm{y}+1 \mathrm{DE}$

Desnutrición leve, entre -1 y -2 DE

Desnutrición moderada, entre -2 y $-3 \mathrm{DE}$

Desnutrición grave, $-3 \mathrm{DE}$

$\begin{array}{rl}3 & 4,4 \% \\ 38 & 55,1 \% \\ 24 & 34,8 \% \\ 4 & 5,8 \% \\ 0 & 0 \%\end{array}$

$p=0,35$

Cuadro 3. Agentes infecciosos identificados.

\begin{tabular}{|c|c|c|c|c|c|}
\hline & \multicolumn{2}{|c|}{ Grupo 1 - SHO/OMS } & \multicolumn{3}{|c|}{ Grupo 2 - Solución de baja osmolaridad } \\
\hline & \# & $\%$ & \# & $\%$ & $\mathbf{p}$ \\
\hline Coprológico & $\mathrm{N}=48$ & & $\mathrm{~N}=47$ & & \\
\hline Ascaris lumbricoides & 2 & 4,2 & 2 & 4,3 & 0,81 \\
\hline Trichuris trichura & 0 & 0 & 1 & 2,1 & 0,25 \\
\hline Giardia lamblia & 1 & 2,1 & 3 & 6,4 & 0,24 \\
\hline Entamoeba histolytica & 0 & 0 & 3 & 6,4 & 0,18 \\
\hline Coprocultivo & $\mathrm{N}=47$ & & $\mathrm{~N}=49$ & & \\
\hline E. coli enteropatógeno & 10 & 21 & 2 & 4,1 & 0,0087 \\
\hline Salmonella enteritidis & 1 & 2,1 & 5 & 10,2 & 0,15 \\
\hline Shigella spp & 0 & 0 & 1 & 2,0 & 0,75 \\
\hline Tinción para Cryptosporidium & $\mathrm{N}=47$ & & $\mathrm{~N}=47$ & & \\
\hline Cryptosporidium parvum & 3 & 6,4 & 0 & 0 & 0,12 \\
\hline Tinción para Campylobacter & $\mathrm{N}=47$ & & $\mathrm{~N}=47$ & & \\
\hline Campylobacter jejuni & 2 & 4,3 & 1 & 2,1 & 0,62 \\
\hline Latex para rotavirus & $\mathrm{N}=47$ & & $\mathrm{~N}=47$ & & \\
\hline Rotavirus positivo & 25 & 53,2 & 16 & 34,0 & 0,067 \\
\hline
\end{tabular}

Cuadro 4. Comportamiento de variables durante el período de hidratación.

\begin{tabular}{|c|c|c|c|c|c|}
\hline & \multicolumn{2}{|c|}{$\begin{array}{l}\text { Grupo } 1 \\
\text { SHO/OMS }\end{array}$} & \multicolumn{2}{|c|}{$\begin{array}{c}\text { Grupo } 2 \\
\text { Solución de baja osmolaridad }\end{array}$} & \multirow[b]{2}{*}{$\mathbf{p}$} \\
\hline & $\mathbf{X}$ & DE & $X$ & DE & \\
\hline Exitos & $61 / 69$ & $88,4 \%$ & $66 / 71$ & $92,9 \%$ & 0.35 \\
\hline Horas de hidratación & 5,2 & 1,83 & 5,5 & 1,7 & 0,31 \\
\hline Suero necesario para hidratación (ml/kg) & 99,4 & 43,3 & 110,4 & 49,1 & 0,33 \\
\hline Suero necesario para hidratación (ml/kg/hora) & 19,5 & 6,9 & 20,2 & 6,4 & 0,50 \\
\hline Gasto fecal (gr/kg/hora) * & 6,3 & 5,0 & 5,6 & 5,1 & 0,94 \\
\hline Peso al ingreso (gr) & 8035,7 & 1627,0 & 8270,0 & 2021,5 & 0,45 \\
\hline Peso al final del periodo de hidratación (gr) & 8431,0 & 1785,0 & 8763,6 & 2148,0 & 0,35 \\
\hline Ganancia de peso (gr) & 404,0 & 231,4 & 486,4 & 261,6 & 0,06 \\
\hline Porcentaje de peso ganado & 4,73 & 2,26 & 5,54 & 2,62 & 0,06 \\
\hline
\end{tabular}

* El gasto fecal durante el período de hidratación incluye todos los pacientes.

Los niveles plasmáticos de sodio al ingreso fueron estadísticamente similares en ambos grupos. Al final del período de hidratación, estas concentraciones plasmáticas fueron diferentes entre los dos grupos $(p=0,013)$. La diferencia se presentó porque el nivel promedio en el grupo 2 fue de 2,6 $\mathrm{mEq} / \mathrm{L}$ menos que en el grupo 1 (cuadro 5).
Por otra parte, al comparar los valores plasmáticos de sodio al ingreso y al final del período de hidratación dentro de cada uno de los grupos, se encontró que en el grupo 1 no hubo diferencias en sus valores promedios $(p=0,38)$, mientras que en el grupo 2 se presentó una disminución de 138,8 $\mathrm{mEq} / \mathrm{L}(\mathrm{DE} 6,3)$ a $136,7 \mathrm{mEq} / \mathrm{L}(\mathrm{DE} 4,3)$. Esta 
Cuadro 5. Electrolitos al comienzo y al final del período de hidratación.

\begin{tabular}{|c|c|c|c|c|c|c|c|}
\hline & \multicolumn{3}{|c|}{$\begin{array}{c}\text { Grupo } 1 \\
\text { SHO/OMS (en } \mathrm{mEq} / \mathrm{L} \text { ) }\end{array}$} & \multicolumn{4}{|c|}{$\begin{array}{c}\text { Grupo } 2 \\
\text { Solución de baja osmolaridad (en } \mathrm{mEq} / \mathrm{L} \text { ) }\end{array}$} \\
\hline & $\mathbf{n}$ & 8 & $\mathrm{DE}$ & $\mathrm{n}$ & 8 & DE & $p$ \\
\hline Sodio inicial & 67 & 139,9 & 8,2 & 70 & 138,8 & 6,3 & 0,38 \\
\hline Sodio al final & 63 & 139,3 & 7,1 & 69 & 136,7 & 4,3 & 0,014 \\
\hline Potasio inicial & 66 & 4,09 & 0,68 & 70 & 4,13 & 0,80 & 0,75 \\
\hline Potasio al final & 65 & 4,40 & 1,25 & 69 & 4,30 & 0,84 & 0,67 \\
\hline
\end{tabular}

diferencia es estadísticamente significativa ( $p=0,0001$ ) (cuadro 5).

En ambos grupos, los niños que presentaron hiponatremia al ingreso normalizaron sus valores durante el período de hidratación.

De seis pacientes que presentaban hipernatremia al ingreso en el grupo 1, tres se normalizaron y tres permanecieron con valores por encima de 150 $\mathrm{mEq} / \mathrm{L}$. Los siete niños que presentaban hipernatremia al ingreso en el grupo 2 tuvieron valores normales al final del período de hidratación.

En ambos grupos hubo dos pacientes que habiendo tenido sodio normal al ingreso, presentaron hiponatremia al final del período de hidratación. En el grupo 1 hubo un niño que habiendo tenido normal el sodio al ingreso, desarrolló hipernatremia leve (153 mEq/L). En el grupo 2 ningún niño desarrolló hipernatremia.

No se presentaron diferencias estadísticas significativas en la concentración plasmática de potasio entre los dos grupos, ni al ingreso ni al final del período de hidratación (cuadro 5).

Dentro de cada grupo se observó un aumento estadísticamente significativo de las concentraciones de potasio durante el período de hidratación (grupo 1, $p=0,035$; grupo 2, $p=0,002$ ).

\section{Periodo de observación}

El promedio del periodo de observación fue 20,9 horas $(D E=5,7)$ en el grupo 1 y 21,9 horas $(D E=5,6)$ en el grupo $2(p=0,28)$.

Hubo ocho niños en el grupo 1 y 2 en el grupo 2 que después de haberse hidratado por vía oral, se deshidrataron de nuevo y fue necesario colocarles líquidos intravenosos antes de completar las primeras veinticuatro horas después del ingreso. Estos niños fueron considerados fracasos tardíos y también fueron excluidos del estudio a partir de ese momento.

Si se suman en cada grupo los niños que en algún momento necesitaron líquidos intravenosos, fueron 16 en el grupo 1 y 7 en el grupo 2, diferencia de frecuencias que fue estadísticamente significativa (riesgo relativo: 1,54 $(1,1-2,15)$, $\mathrm{p}=0,033$ ).

De los 117 niños restantes, en quienes se consiguió con éxito la hidratación y no se presentó recurrencia en el estado de deshidratación, el periodo de observación fue de veinticuatro horas en 108 casos. En nueve casos, los niños tuvieron que ser dados de alta antes de cumplir las veinticuatro horas del ingreso porque los padres tenían dificultades para permanecer en el servicio.

Los datos de todos los 140 pacientes fueron incluidos en el análisis hasta el momento en que participaron en el estudio.

Como el periodo de observación no fue igual para todos los niños, los resultados no se presentan en términos absolutos sino considerando el número de horas durante las cuales cada niño permaneció en el estudio.

El gasto fecal durante el período de observación fue $5,2(\mathrm{DE}=4,1) \mathrm{g} / \mathrm{kg} / \mathrm{hora}$ en el grupo 1 y 4,2 $(D E=4,1) \mathrm{g} / \mathrm{kg} / \mathrm{hora}$ en el grupo 2 ; la diferencia no fue estadísticamente significativa $(p=0,16)$ (cuadro 6).

No se presentaron diferencias estadísticas significativas en relación con la ingestión de suero, el consumo de fórmula y de sopa, la eliminación urinaria y el volumen de los vómitos y número de vómitos durante el período de observación. 
Tampoco se presentaron diferencias en relación con la cantidad de niños que recibieron leche materna durante este período (cuadro 6).

Los 18 niños que presentaban diarrea persistente, nueve en cada grupo, lograron hidratarse satisfactoriamente. En el grupo 1, dos de los niños con diarrea persistente se deshidrataron de nuevo.

Entre los siete niños que presentaban sangre en las heces en el grupo 1, se presentó un fracaso tardío. De los 15 niños con diarrea con sangre en el grupo 2, hubo dos fracasos tempranos.

No se presentaron diferencias estadísticas significativas en relación con el número de deposiciones durante el período de observación.

Por efecto del azar se presentaron 10 pacientes que tenían E. colienteropatógeno en el grupo 1 y solamente 2 en el grupo 2, esta diferencia fue significativa $(p=0,009)$, lo que de alguna manera podría hacer que los grupos no fueran estrictamente comparables. Se analizó el gasto fecal durante el período de observación en cada grupo, entre los niños a quienes se pudo hacer coprocultivo, estudiando por separado los que tenían E. coli y los que no lo tenían. En los 10 pacientes que tenían $E$. colien el grupo 1 , el gasto fecal durante el período de observación fue 5,73 $\mathrm{g} / \mathrm{kg} / \mathrm{hora}(\mathrm{DE}=5,74)$; en los 38 pacientes del grupo 1 en quienes se pudo hacer coprocultivo y no se encontró $E$. coli, el gasto fecal fue $6,21 \mathrm{~g} / \mathrm{kg} / \mathrm{hora}$
$(\mathrm{DE}=4,4)$; esta diferencia no fue significativa $(p=0,78)$. De igual manera, en los dos pacientes que tenían $E$. coli en el grupo 2, el gasto fecal durante el período de observación fue $5,83 \mathrm{~g} / \mathrm{kg} /$ hora $(D E=7,25)$ y entre los 47 pacientes a quienes se les hizo coprocultivo en este grupo y no tenían E. coli, el gasto fecal fue $3,89 \mathrm{~g} / \mathrm{kg} / \mathrm{hora}(\mathrm{DE}=2,50)$, esta diferencia tampoco fue significativa $(p=0,77)$. De esta manera se pudo mostrar que la mayor presencia de $E$. coli en el grupo 1 no explicó el mayor gasto fecal en ese grupo.

\section{Discusión}

El hallazgo más destacado de este estudio fue que los niños que recibieron la SBO tuvieron un riesgo significativamente menor de necesitar líquidos intravenosos durante las veinticuatro horas siguientes al inicio de la hidratación oral. Esta es una ventaja importante que puede significar menores costos, menos trauma y menor necesidad de hospitalizar a los pacientes que han presentado recurrencia en el estado de deshidratación. En el estudio multicéntrico (12) en el que se empleó una solución con $60 \mathrm{mmol} / \mathrm{L}$ de sodio, el riesgo de necesitar líquidos intravenosos fue mayor entre los niños que recibieron el SHO/90, aun cuando la diferencia no fue significativa, $R R=1,4(0,9-2,4)$. En el estudio de Santosham et al. (14), que utilizó la misma solución empleada en el presente estudio, también se encontró un mayor riesgo de necesitar

Cuadro 6. Comportamiento de diferentes parámetros durante el período de observación en cada uno de los grupos.

\begin{tabular}{|c|c|c|c|c|c|}
\hline & \multicolumn{2}{|c|}{$\begin{array}{l}\text { Grupo } 1 \\
\text { SHO/OMS }\end{array}$} & \multicolumn{2}{|c|}{$\begin{array}{c}\text { Grupo } 2 \\
\text { Solución de baja osmolaridad }\end{array}$} & \multirow[b]{2}{*}{$\mathbf{p}$} \\
\hline & $\mathbf{x}$ & DE & $\mathbf{x}$ & DE & \\
\hline Tiempo de observación en horas & 21 & 5,7 & 22 & 5,6 & 0,31 \\
\hline Gasto fecal $(\mathrm{gr} / \mathrm{kg} / \mathrm{h})$ & 5,2 & 4,1 & ,2 & 4,1 & 0,16 \\
\hline Número de deposiciones $(\mathrm{n})$ & 10,0 & 5,2 & 9,2 & 5,5 & 0,37 \\
\hline Suero ingerido $(\mathrm{ml} / \mathrm{kg} / \mathrm{h})$ & 9,3 & ,6 & 8,5 & 6,4 & 0,47 \\
\hline Volumen de fórmula $(\mathrm{ml} / \mathrm{g} / \mathrm{h})$ & 2,4 & 1,2 & 2,3 & 1,2 & 0,64 \\
\hline Volumen de sopa $(\mathrm{ml} / \mathrm{kg} / \mathrm{h})$ & 0,6 & 0,7 & 0,6 & 0,7 & 0,99 \\
\hline Recibieron leche materna $(n)$ & $15(21,7 \%)$ & & $17(23,9 \%)$ & & 0,77 \\
\hline Número de tomas de leche materna $(n)$ & 3,13 & 2,55 & 4,17 & 2,57 & 0,26 \\
\hline Eliminación urinaria $(\mathrm{ml} / \mathrm{kg} / \mathrm{h})$ & 1,1 & 1,0 & 1,3 & 1,0 & 0,29 \\
\hline Volumen de vómito ( $\mathrm{gr} / \mathrm{kg} / \mathrm{h})$ & 0,3 & 0,4 & 0,5 & 1,6 & 0,31 \\
\hline Número de vómitos $(\mathrm{n})$ & 2,0 & 2,4 & 1,9 & 2,0 & 0,68 \\
\hline Peso al final del periodo de observación (gr) & 8401,1 & 1754,3 & 8685,7 & 2103,2 & 0,41 \\
\hline Ganancia de peso al final del paeriodo (gr) & 374,2 & 250,7 & 408,6 & 290,5 & 0,48 \\
\hline Porcentaje de peso ganado al final & 4,46 & 2,78 & 4,78 & 3,22 & 0,55 \\
\hline
\end{tabular}


líquidos parenterales entre los niños que recibieron el SHO/90, en este caso la diferencia tampoco alcanzó a ser significativa: $R R=2,8(0,9-8,4)$.

En el presente estudio, el gasto fecal en los niños que recibieron la SBO fue $11 \%$ menor durante la fase de la hidratación. Esta disminución no alcanzó a ser estadísticamente significativa. En el estudio de Santosham et al. (14), en el que se utilizó la misma solución, se encontró gasto fecal 36\% menor durante el período de hidratación entre los niños que recibieron la SBO; esta reducción sí fue estadísticamente significativa. En dicho estudio, como en el nuestro, no hubo diferencias significativas en el tiempo necesario para conseguir la hidratación, en el porcentaje de peso ganado ni en la cantidad de suero necesaria para conseguir la hidratación.

Los resultados obtenidos fueron satisfactorios en relación con el comportamiento del sodio plasmático con la SBO. A pesar de que, en promedio, los valores del sodio plasmático disminuyeron, se corrigieron todos los casos de hiponatremia y de hipernatremia y solamente dos pacientes que tenían sodio normal al ingreso resultaron al final de la hidratación con valores por debajo de $130 \mathrm{mEq} / \mathrm{L}$, lo cual ocurrió también en dos de los niños que recibieron el SHO/90. En el estudio multicéntrico (12) realizado con una solución con una concentración de sodio de 60 $\mathrm{mmol} / \mathrm{L}$, el riesgo de desarrollo o empeoramiento de la hiponatremia fue ligeramente mayor, aun cuando no en forma significativa, entre los niños que recibieron la solución experimental.

La concentración de $75 \mathrm{mmol} / \mathrm{L}$ en una solución de hidratación oral es suficientemente segura para la hidratación de los niños con diarrea no colérica y no genera casos de hiponatremia. Hallazgos similares se encontraron en el estudio de Santosham et al. (14).

Ambas soluciones contienen la misma concentración de potasio y el comportamiento del potasio plasmático fue similar en los dos grupos.

El periodo de observación establecido para el presente estudio comprendió las veinticuatro horas siguientes al ingreso, durante este período se incluyeron también las horas utilizadas para conseguir la hidratación. Se consideró que deberían incluirse para el análisis todos los niños, incluidos los fracasos tanto tempranos o tardíos durante el tiempo que recibieron el suero oral. Si los fracasos hubieran sido excluidos y se hubiera considerado el gasto fecal solamente entre los que se hidrataron y continuaron recibiendo el suero oral, probablemente se hubieran excluido del análisis precisamente aquellos niños con gasto fecal más alto. En este estudio, el gasto fecal durante el período de observación se expresa en $\mathrm{g} / \mathrm{kg} / \mathrm{hora}$, ya que el análisis se hizo durante las horas en que cada paciente recibió el suero oral.

El gasto fecal durante el período de observación fue $19 \%$ menor entre los niños que recibieron la SBO; sin embargo, esta diferencia no fue significativa. En el estudio multicéntrico (12), en el cual se utilizó un suero con $60 \mathrm{mmol} / \mathrm{L}$ de sodio, el gasto fecal durante las primeras veinticuatro horas sí fue significativamente menor entre los niños que recibieron la SBO. En el estudio de Santosham (14) no se encontraron diferencias estadísticas significativas en relación con el gasto fecal durante las veinticuatro horas que siguieron al momento en que se consiguió la hidratación.

En los estudios anteriores sólo se incluyeron niños con diarreas acuosas y de menos de cinco días de evolución. En este estudio se incluyeron niños con diarrea con sangre y con diarrea persistente. Cuando se programó el estudio se consideró que tanto los niños con diarrea con sangre, como los que tienen diarrea persistente, si están deshidratados, deben hidratarse por vía oral; por tanto, un suero de hidratación oral debe ser lo suficientemente efectivo para hidratar niños deshidratados por diarreas agudas, persistentes o disentéricas. No hubo diferencias en la proporción de éxitos y fracasos en relación con el tipo de diarrea.

Con este estudio se concluyó que tanto el $\mathrm{SHO} /$ 90 como la SBO son muy efectivos y seguros para tratar la mayoría de los niños deshidratados por enfermedades diarreicas. La utilización de la SBO disminuyó el riesgo de que fuera necesario el uso de líquidos intravenosos durante las primeras veinticuatro horas de iniciada la hidratación. 
Después de la publicación original de este estudio, se han dado otras publicaciones y hechos importantes que deben tenerse en cuenta.

En abril de 2001, se publicó un nuevo estudio multicéntrico, realizado con el apoyo de la OMS, para evaluar la eficacia de la solución de baja osmolaridad con la misma composición utilizada en el presente estudio (21). Se incluyeron 675 niños de uno a veinticuatro meses con deshidratación secundaria a diarrea aguda. Se encontró, como en nuestro estudio, una menor proporción de niños que necesitaron líquidos intravenosos entre los que recibieron la solución de baja osmolaridad (10\%), comparado con el grupo de niños que recibió SHO/90 (15\%) OR=0,6 (IC95\%: 0,4-1). Tampoco encontraron diferencias en relación con el gasto fecal entre los niños que recibieron la SBO en comparación con los que recibieron $\mathrm{SHO} / 90$ ni durante las primeras veinticuatro horas, ni durante todo el tiempo de duración de la diarrea. No hubo diferencias significativas en relación con la incidencia de hiponatremia a las 24 horas entre los dos grupos de tratamiento. La frecuencia de pacientes con sodio sérico menor de $125 \mathrm{mmol} / \mathrm{L}$ a las 24 horas fue de $4 \%$ entre los niños que recibieron la SBO y de $2 \%$ entre quienes recibieron $\mathrm{SHO} / 90$. Uno de los niños que recibió la solución de baja osmolaridad y que tenía hiponatremia presentó una convulsión de corta duración. Este niño, además, tenía fiebre.

En julio de 2001 se publicó un metanálisis en el que se incluyeron 15 estudios controlados, incluido el que es objeto de esta publicación (22). Se compararon las soluciones de baja osmolaridad con concentraciones de sodio entre 50 y $75 \mathrm{mmol} /$ $\mathrm{L}$ y osmolaridad entre 210 y $268 \mathrm{mOsm} / \mathrm{L}$ con $\mathrm{SHO} / 90$. La variable principal que se tuvo en cuenta en el metanálisis fue la necesidad de utilizar líquidos intravenosos durante el tratamiento; se analizaron también la reducción del gasto fecal y la presencia de hiponatremia. El uso de la SBO redujo en $35 \%$ la necesidad de utilizar líquidos intravenosos, OR 0,61 (IC 95\% $0,47-0,81$ ), resultados muy similares a los del presente estudio. La reducción del gasto fecal cuando se utilizaron las SBO fue $20 \%$, la diferencia de medias del gasto fecal en escala logarítmica fue estadísticamente significativa, $-0,214$ (IC 95\% -0,305 a -0,123). No hubo diferencia estadística significativa en la incidencia de hiponatremia, aunque fue mayor entre los niños que recibieron la solución de baja osmolaridad, OR=1,45 (IC95\% 0,93 a 2,26).

En el mismo año, se realizó una reunión de expertos citada por el Fondo de las Naciones Unidas para la Infancia (Unicef) y la OMS con el objetivo de revisar los resultados de las investigaciones anteriores y las más recientes, para dar recomendaciones técnicas sobre la seguridad y la eficacia de las soluciones de baja osmolaridad en adultos y niños con cólera y en niños con diarrea aguda no colérica (23).

En relación con la eficacia de las soluciones de baja osmolaridad en adultos con cólera, el grupo de expertos revisó los estudios disponibles y concluyó que la SBO con $75 \mathrm{mmol} / \mathrm{L}$ de sodio es tan efectiva como la SHO/90. Sin embargo, señalan que se presentó una reducción de los valores de sodio plasmático de $1,3 \mathrm{mEq} / \mathrm{L}$ (IC 95\%: 0,3 a 2,3 ), que, aun cuando no fue estadística-mente significativa, sugiere la monitorización clínica y bioquímica cuando la solución de baja osmolaridad sea introducida para uso rutinario. En los estudios sobre niños con cólera los pacientes incluidos son pocos; sin embargo, el análisis del grupo de expertos permitió concluir que el suero de baja osmolaridad es tan efectivo como el SHO/90. No hubo diferencia estadística significativa en el riesgo de contraer hiponatremia, aunque fue mayor con las sales de baja osmolaridad RR de 1,8, (IC95\%: 0,9 a 3,2); de todas maneras se sugiere una monitorización cuidadosa sobre la concentración plasmática de sodio en el uso rutinario de soluciones de baja osmolaridad en estos pacientes.

En los niños con diarrea aguda no colérica, el grupo de expertos hizo un nuevo análisis de los estudios que evaluaron los efectos del suero de baja osmolaridad en el sodio plasmático, estratificándolos de acuerdo con la concentración de sodio de las soluciones, se agruparon las que tenían $75 \mathrm{mmol} / \mathrm{L}$ de sodio y los que tenían menos. Se encontró disminución del gasto fecal estadísticamente significativa en los ocho 
estudios en los cuales la concentración de sodio era menor de 75 , pero en los cuatro que utilizaron exactamente 75 no hubo reducción del gasto fecal estadísticamente significativa. En este mismo análisis estratificado se encontró que las soluciones con exactamente $75 \mathrm{mmol} / \mathrm{L}$ de sodio no conducen a un mayor riesgo de hiponatremia que la SHO/90, OR=1,45 (IC 95\%: 0,93 a 2,26). EI nuevo análisis confirmó la disminución de la necesidad de utilizar líquidos intravenosos con las soluciones de $75 \mathrm{mmol} / \mathrm{L}$.

El comité de expertos recomendó mantener la política de una solución única para prevenir y tratar la deshidratación producida por diarreas de cualquier etiología y que esta solución contenga $75 \mathrm{mmol} / \mathrm{L}$ de sodio, $75 \mathrm{mmol} / \mathrm{L}$ de glucosa y una osmolaridad de $245 \mathrm{mOsm} / \mathrm{L}$.

En mayo de 2002, la Asamblea de la OMS cambió oficialmente la formulación de las sales de hidratación oral por las de baja osmolaridad.

Algunos expertos (24-26) han manifestado su inconformidad con este cambio, para ellos el nuevo suero no llena las expectativas del 'súper suero', ya que finalmente no disminuye el volumen ni la duración de la diarrea y la ventaja de reducir la necesidad de utilizar líquidos intravenosos la consideran marginal. Por otra parte, creen que la nueva fórmula no es suficientemente segura para evitar la hiponatremia y sugieren la necesidad de realizar más estudios. Sin embargo, el modelo de análisis para la toma de decisiones utilizado por el grupo de expertos de la OMS, que evalúa los beneficios del cambio en la formulación, considera que las sales de hidratación oral de baja osmolaridad evitarán 14.000 muertes por millón de episodios de diarrea con algún grado de deshidratación al reducir el número de fallas en el tratamiento, lo que tendría un mayor peso que la posibilidad de ocurrencia de convulsiones debidas a hiponatremia.

\section{Agradecimientos}

El estudio fue financiado por el Instituto Colombiano para el Desarrollo de la Ciencia y Tecnología, Francisco José de Caldas, Colciencias (1115-04-348-96), la Universidad de Antioquia, el Hospital Universitario San Vicente de Paúl y la Corporación de Fomento al Hospital San Vicente de Paúl, Corpaúl.

\section{Referencias}

1. The International Study Group on Improved ORS. Impact of glycine-containing ORS solutions on stool output and duration of diarrhoea: a meta-analysis of seven clinical trials. Bull WHO 1991;69:541-8.

2. Gore SM, Fontaine O, Pierce NF. Impact of rice based oral rehydration solution on stool output and duration of diarrhoea: meta-analysis of 13 clinical trials. BMJ 1992; 304:287-91.

3. Fayad IM, Hashem M, Duggan C. Comparative efficacy of rice-based and glucose-based oral rehydration salts plus early reintroduction of food. Lancet 1993;342:772-5.

4. Kenya PR, Odongo HW, Oundo G. Cereal based oral rehydration solutions. Arch Dis Child 1989;64:10325.

5. Bhan MK, Ghai OP, Khoshoo V. Efficacy of mung bean (lentil) and pop rice based rehydration solutions in comparison with the standard glucose electrolyte solution. J Pediatr Gatroenterol Nutr 1987;6:392-9.

6. Alam AN, Sarker SA, Molla AM, Rahaman MM, Greenough WB. Hydrolysed wheat based oral rehydration solution for acute diarrhoea. Arch Dis Child 1987;62:440-4.

7. Santos Ocampo PD, Bravo IC, Rogacion JM, Battad GR. A randomized double-blind clinical trial of a maltodextrin-containing oral rehydration solution in acute infantile diarrhea. J Pediatr Gastroenterol Nutr 1993;16: 23-8.

8. Sack RB, Castrellon J, Della Sera E. Hydrolyzed lactalbumin-based oral rehydration solution for acute diarrhoea in infants. Acta Paediatr 1994;83:819-24.

9. Lebenthal E, Khin-maung-U, Rolson DDK, KhinMyat-Tun, Tin-Nu-Swe, Tthein-Thein-Myint, et al. Termophilic amylase-digested rice-electrolyte solution in the treatment of acute diarrhea in children. Pediatrics 1995; $95: 198-202$.

10. Arias MM, Alcaraz GM, Bernal C, González G. Oral rehydration with a plantain-flour based solution in children dehydrated by acute diarrhea: A clinical trial. Acta Paediatr 1997;86:1047-51.

11. El-Mougi M, El-Akkad N, Hendaw A, Hassan M, Amer A, Fontaine O, Pierce N.F. Is a low-osmolarity ORS solution more efficacious than standard WHO ORS solution? J Ped Gastroenterol Nutr 1994;19:83-6.

12. International Study Group on Reduced-Osmolarity ORS Solutions. Multicentre evaluation of reducedosmolarity oral rehydration salts solution. Lancet 1995; 345:282-5.

13. World Heath Organization. 25 years of ORS. Joint WHO/ICDDR,B Consultative meeting on OR formulation. 
Dhaka, Bangladesh, 10-12 December, 1994. WHO/ CDR/CDD/95.2. Geneva: WHO; 1994.

14. Santosham M, Fayad I, Ziki M, Hussein A, Amponsah A, Duggan C. A double-blind clinical trial comparing World Health Organization oral rehydration solution with a reduced osmolarity solution containing equal amounts of sodioum and glucose. J Ped 1996;128: 45-51.

15. Bernal C, Velásquez C, García G, Palacio C, Uribe G. Hidratación oral con una solución de baja osmolaridad. Saludarte 2000;1:6-23.

16. Pocock SJ. Clinical trials. A practical approach. Chichester: John Wiley \& Son; 1987. p.266.

17. OPS/OMS. Manejo del paciente con diarrea. Programa de control de la enfermedades diarréicas. Tercera Edición. Washington, D.C.: OPS; 1991.

18. World Health Organization. Programme of control of diarrhoeal diseases. Manual for laboratory investigations of acute enteric infections. CDD/83.3 Rev.1. Geneve: WHO; 1987.

19. Bernal C. Enfermedad diarreica y Deshidratación. En: Gómez JF, Gómez LF, González D, editores. Pautas de tratamiento en pediatría. Tercera edición. Medellín: Editorial Universidad de Antioquia; 1997. p.106-41.

20. Hamil PW, Drizid TA, Johnson CL, Reed RB, Roche AF, Moore WM. Physical growth: National Center for
Health Statistics Percentiles. Am J Clin Nutr 1979; 32:607-29.

21. CHOICE Study Group. Multicenter randomized double blind clinical trial to evaluate the efficacy and safety of a reduced osmolarity oral rehydration salts solution in children with acute watery diarrhea. Pediatrics 2000; 107:613-8.

22. Hahn SK, Kim YJ, Garner P. Reduced osmolarity oral rehydration solution for treating dehydration due to diarrhoea in children: systematic review. BMJ 2001;323: 81-5.

23. World Heath Organization. Reduced osmolarity oral rehydration salts (ORS) formulation. Report from a meeting of experts jointly organized by UNICEF and WHO. New York, USA, 18 july, 2001. WHO/FCH/CAH/ 01.22. Geneva: WHO; 2001.

24. Fuchs GJ. Reduced osmolarity rehydration solutions: new and improved ORS? J Pediatr Gastroenterol Nutr 2002;34:252-3.

25. Hirschhorn N, Nalin DR, Cash RA. CHOICE Study Group trial. Pediatrics 2002;109:713-5.

26. Hirschhorn N, Nalin DR, Cash RA, Greenough III WB. Formulation of oral rehydration solution. Lancet 2002; 360:340-1. 